\title{
The Lunar Atmosphere and Dust Environment Explorer Mission
}

\author{
Butler Hine, Stevan Spremo, Mark Turner \\ NASA Ames Research Center \\ Mail Stop 240-5 \\ Moffett Field, CA 94035 \\ 650-604-4449 \\ butler.p.hine@nasa.gov \\ Robert Caffrey \\ NASA Goddard Space Flight Center \\ Mail Stop 401.0 \\ Greenbelt, MD 20771 \\ 301-286-0846 \\ robert.t.caffrey@nasa.gov
}

Abstract - The Lunar Atmosphere and Dust Environment Explorer (LADEE) is a Lunar science orbiter mission currently under development to address the goals of the National Research Council decadal surveys and the recent "Scientific Context for Exploration of the Moon" (SCEM) [1] report to study the pristine state of the lunar atmosphere and dust environment prior to significant human activities. ${ }^{12}$ LADEE will determine the composition of the lunar atmosphere and investigate the processes that control its distribution and variability, including sources, sinks, and surface interactions. LADEE will also determine whether dust is present in the lunar exosphere, and reveal the processes that contribute to its sources and variability. These investigations are relevant to our understanding of surface boundary exospheres and dust processes throughout the solar system, address questions regarding the origin and evolution of lunar volatiles, and have potential implications for future exploration activities. The LADEE science instruments include a neutral mass spectrometer, ultraviolet spectrometer, and dust sensor. LADEE will also fly a laser communications system technology demonstration that could provide a building block for future space communications architectures. LADEE is an important component in NASA's portfolio of near-term lunar missions, addressing objectives that are currently not covered by other U.S. or international efforts, and whose observations must be conducted before large-scale human or robotic activities irrevocably perturb the tenuous and fragile lunar atmosphere. LADEE will also demonstrate the effectiveness of a low-cost, rapid-development program utilizing a modular bus design launched on the new Minotaur V launch vehicle. Once proven, this capability could enable future lunar missions in a highly cost constrained environment. This paper describes the LADEE objectives, mission design, and technical approach.

\footnotetext{
${ }^{1}$ U.S. Government work not protected by U.S. copyright

${ }^{2}$ IEEEAC paper \#1285, Version 7, Updated January 4, 2010
}

\section{TABLE OF CONTENTS}

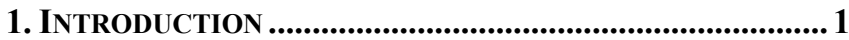

2. MISSION REQUIREMENTS..................................................... 2

3. MISSION DESIGN........................................................... 2

4. SPACECRAFT DESIGN .............................................................. 5

5. MISSION SYSTEM ARCHITECTURE...................................... 8

6. LAUNCH VEHICLE ................................................................. 9

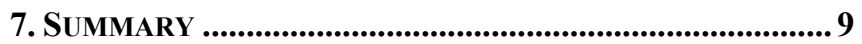

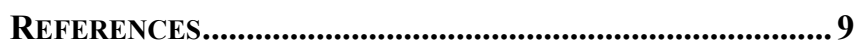

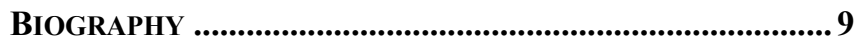

\section{INTRODUCTION}

National Research Council decadal surveys and the recent "Scientific Context for Exploration of the Moon" (SCEM) report [1] identify studies of the pristine state of the lunar atmosphere and dust environment as among the leading priorities for future lunar science missions. The Lunar Atmosphere and Dust Environment Explorer (LADEE) Mission was created in March 2008 to address these goals. LADEE will determine the composition of the lunar atmosphere and investigate the processes that control its distribution and variability, including sources, sinks, and surface interactions. LADEE will also determine whether dust is present in the lunar exosphere, and reveal the processes that contribute to its sources and variability. These investigations are relevant to our understanding of surface boundary exospheres and dust processes throughout the solar system, address questions regarding the origin and evolution of lunar volatiles, and have potential implications for future exploration activities.

LADEE employs a high-heritage science instrument payload, including a neutral mass spectrometer, an ultraviolet spectrometer, and an in-situ dust sensor. In addition to these science instruments, LADEE will also carry a laser communications system technology demonstration. These instrument payloads are more fully described in an accompanying paper in this conference [2]. 
The LADEE spacecraft bus design was derived from the Modular Common Spacecraft Bus (MCSB, a.k.a "Common Bus") architecture developed at NASA Ames Research Center (ARC) from 2006-2008 [3]. The MCSB is a small, low-cost spacecraft designed to deliver scientifically and technically useful payloads to a variety of locations, including low earth orbit (LEO), lunar orbit and lunar surface, earth-moon lagrange points, and near earth objects (NEOs). The spacecraft bus is an innovative modular bus design applicable to a wide range of missions, including orbiters and landers. The original design objectives were that the missions fit within a budget of $\$ 100$ Million, not including science instruments, and could be launched on a variety of affordable launch vehicles such as the MinotaurV or Delta II. The key advantages of smaller spacecraft such as this are reduced costs and rapid development schedules.

LADEE is an important component in NASA's portfolio of near-term lunar missions, addressing objectives that are currently not covered by other U.S. or international efforts, and whose observations must be conducted before largescale human or robotic activities irrevocably perturb the tenuous and fragile lunar atmosphere. LADEE's success will also demonstrate the effectiveness of a low-cost, rapid development program, utilizing a modular bus design together with the new Minotaur V launch vehicle, and will thus pave the way for future lunar missions in a costconstrained environment.

\section{MISSION REQUIREMENTS}

The top-level programmatic and science requirements for the LADEE project are designed to accomplish the following mission objectives:

1) Determine the composition of the lunar atmosphere and investigate the processes that control its distribution and variability, including sources, sinks, and surface interactions.

2) Characterize the lunar exospheric dust environment and measure any spatial and temporal variability and impacts on the lunar atmosphere

3) Demonstrate the Lunar Laser Com Demonstration (LLCD)

4) Create a low-cost reusable spacecraft architecture that can meet the needs of certain planetary science missions

5) Demonstrate the capability of the Minotaur V as a launch vehicle for planetary missions

In order to meet the first two objectives, the spacecraft will carry three instruments to conduct in-situ observations: a Neutral Mass Spectrometer (NMS), an Ultra Violet Spectrometer (UVS), and the Lunar Dust Experiment (LDEX). The mission will use a retrograde near-equatorial science orbit, take measurements at low altitude $(<50 \mathrm{~km})$, and capture measurements over at least one month in duration.

The mission is designed to capture all required science measurements within the first 100 days after orbit/commissioning. Currently, the science instruments and LLCD operations are decoupled in that the LLCD operations are accomplished during the commissioning phase. Thus, the 100 days of science operations begin after the LLCD technology demonstration and commissioning activities are finished, and the spacecraft has transferred to the appropriate altitude to begin science operations.

\section{Mission Design}

The LADEE Mission will consist of five major phases: prelaunch, launch and early mission, commissioning, nominal operations (e.g. science operations), and extended mission/end-of-life.

The satellite is expected to launch from The NASA GSFC Wallops Flight Facility (WFF) in late 2012. Once launched, the spacecraft will perform several phasing loops and then arrive at the Moon where it will establish an elliptical orbit of approximately $250 \times 75 \mathrm{~km}$. The satellite will perform a 30 day checkout and commissioning phase, during which time it will also perform the LLCD technology demonstration. Once finished, the spacecraft will drop to a lower science orbit $(\sim 50 \mathrm{~km})$ where it will perform nominal science operations. The science phase will last 100 days. After 100 days, the mission will continue for as long as it has consumables (e.g. propellant), and then will be sent into a guided crash into the Moon. Figure 1 shows a schematic view of the various mission phases.

\section{Launch, Ascent, Separation, Acquisition}

Launch will take place from the Wallops Flight Facility aboard a Minotaur V launch vehicle. Preliminary analysis of launch windows indicates opportunities of approximately 3-5 days per month with optimum launch windows of about 15 minutes.

\section{LTO, Phasing Loops, and TCMs}

The lunar transfer orbit (LTO) uses a phasing loop design where the launch vehicle places the spacecraft on a trajectory with a maximum altitude on the order of 50 Earth radii (in comparison to the Moon's altitude, which is about 60 Earth radii). In the current design, the spacecraft orbits the Earth three times in this highly elliptical orbit, making trajectory corrections on each pass if necessary (at perigee and/or apogee). On the final perigee, the onboard propulsion subsystem will add enough energy to the orbit for the final apogee to occur where the Moon's gravity can take over, beginning the hyperbolic approach trajectory at the Moon. 
On approximately three to four occasions during the 25-day cruise phase, the vehicle will reorient to align the thrusters with the velocity vector (in the same direction to add energy or in the opposite direction to decrease orbit energy). For a large maneuver carried out with small thrusters, it is possible for the maneuver duration to approach a large fraction of an hour because of thruster duty cycles and temperature constraints. The procedure for executing maneuvers during cruise will have the following basic steps: stop rotation, slew to burn attitude, fire thrusters, slew back to cruise attitude, resume rotation. During the burn, the

\section{Lunar Orbit Insertion (LOI)}

After rendezvous with the Moon's gravitational field, the spacecraft will disappear behind the Moon on its descent to minimum altitude. When it re-emerges around the limb of the Moon, it will execute a two-burn Lunar LOI approach. The thrust vector will be opposite the velocity vector. Communications direction to the Earth will be very near the velocity vector and the plume direction. The burn time is expected to be approximately 10 minutes. After the burns, the spacecraft will be at a relatively high $(250+\mathrm{km})$ circular

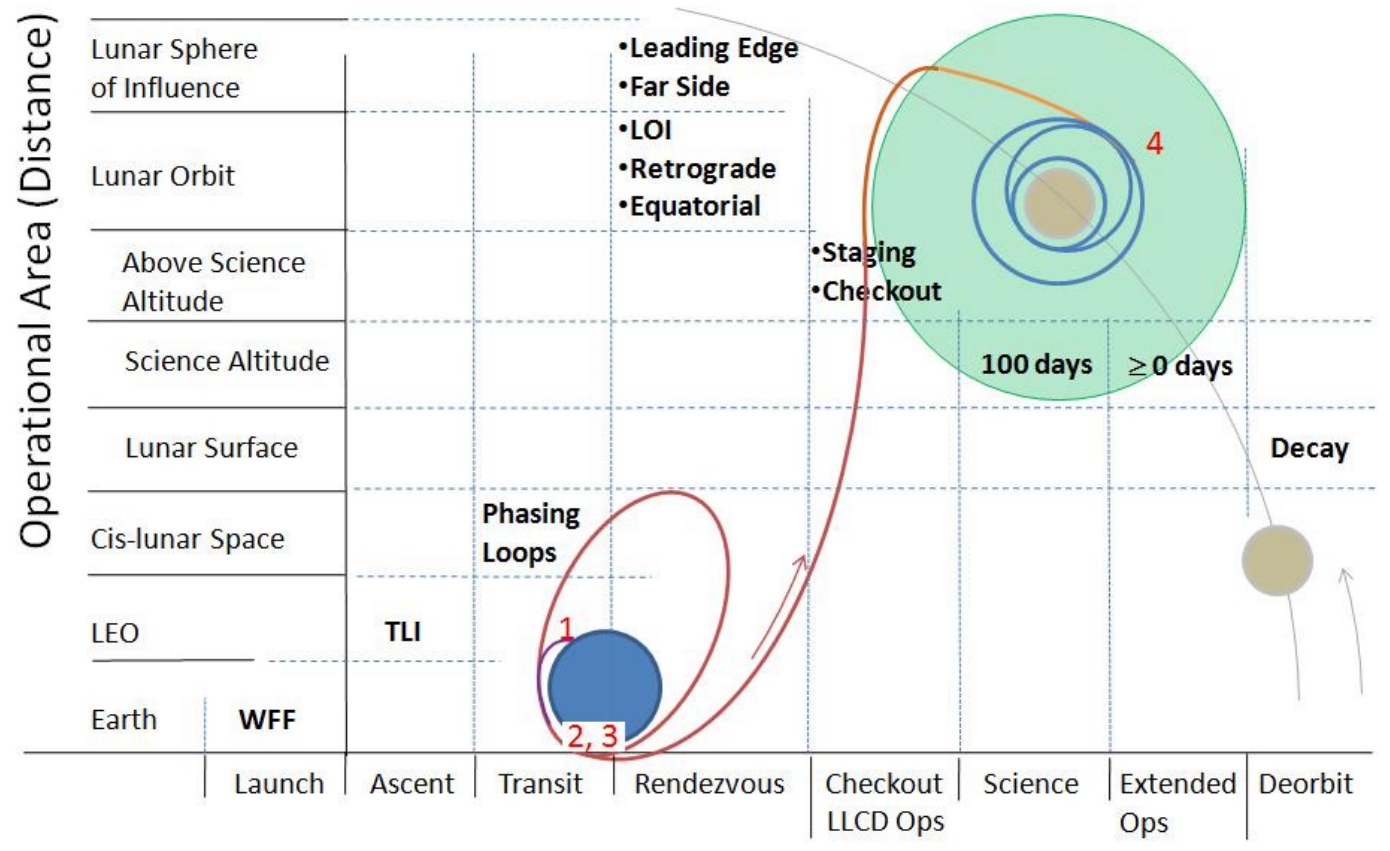

Mission Phase

Af 1 Launch: $\quad$ 2TLI: $3 \mathrm{TCM}(\mathrm{s}): \quad 4$ LOI:

Figure 1: Schematic representation of the LADEE mission phases

optimum attitude will change slightly because of the arc of the trajectory. Three-axis stabilization will probably be used during maneuvers.

During the phasing loops, the payload instruments (including the LLCD Lunar Lasercom Space Terminal (LLST)) will be powered on to check the electronics. The instrument apertures will remain covered due to concerns over contamination from the lunar orbit insertion (LOI) burn and the composite structure outgassing, but aliveness tests can be performed. The LLCD is interested in running operational tests during the phasing loops because the close proximity to Earth ensures a strong signal and the spacecraft will also be producing more available power, which would potentially allow the LLST to operate for a longer period of time. orbit.

\section{Initial Checkout/Commissioning}

The commissioning phase will last 30 days, during which the LLCD will carry out its technology demonstration. The checkout and commissioning activity of the science instruments will be carried out during this phase as well, following the initial aliveness tests performed during the phasing loops.

The Commissioning Phase orbits will start at an altitude higher than nominal science operations. The initial orbit after the LOI will be approximately $250 \mathrm{~km}$ circular, but within a couple of days a transfer to $75 \mathrm{~km}$ periapsis will be used to establish the orientation of the periapsis over the sunrise terminator. The resulting $75 \times 250 \mathrm{~km}$ orbit will be allowed to degrade throughout most of the commissioning phase, eventually reaching approximately 40 x $300 \mathrm{~km}$. 
There will be few (if any) orbit maintenance maneuvers during this phase. There will be, however, 3-axis attitude maneuvers for pointing the spacecraft, which will be commanded by Mission Operations.

Some time after orbit insertion, the spacecraft will uncover the science instruments and perform checkout on each one. Calibration activities will be lighter than science operations, with the duty cycle for the instruments being approximately half of a science orbit. Primary interest will focus on operating the science instruments at periapsis when the spacecraft is at low altitudes, which will be more similar to Science Operations.

\section{Lunar LASER Operations/Ongoing Commissioning}

The LLCD will be operated during the Commissioning Phase after the three science instruments have been commissioned. A LLCD orbit will include 13 to 15 minutes "on" time of the LLST with no science instruments. Power concerns may limit the number of LLCD orbits that can run in sequence before a dedicated orbit for recharging the batteries must occur. During the technology demonstration, the spacecraft must be pointed at the Earth with an accuracy error of $<1$ degree.

\section{Orbit Transfer}

The spacecraft will perform a Hohmann transfer to go from the commissioning orbit to the operational science orbit. The vehicle will slew to align the thrust vector with the velocity delta, execute the delta- $\mathrm{V}$ maneuver, and go to the preferred post-maneuver attitude. If communications, power, or thermal considerations require it, the postmaneuver attitude may require another slew. If the second portion of the Hohmann transfer occurs right away, there may be no reason not to stay in the maneuver attitude until the final orbit is reached. Upon arrival in the operational orbit, the vehicle will assume its standard, 3-axis stabilized orientation with slow spin about the longitudinal axis for thermal control.

\section{Science Operations}

At the beginning of this phase, the spacecraft will be at an altitude and alignment compatible with science operations. The LADEE science requirements specify that low-altitude passes of $<50 \mathrm{~km}$ occur over the sunrise terminator of the Moon. The unevenness of the Moon's gravitational field requires a significant amount of delta-V $(\sim 400 \mathrm{~m} / \mathrm{s})$ to maintain a $50 \mathrm{~km}$ orbit for 100 days. To minimize spacecraft mass/complexity it was decided to use an elliptical orbit with apoapsis in the range of 60 to $80 \mathrm{~km}$ and periapsis between 20 and $50 \mathrm{~km}$ that satisfies the science requirements. This class of orbit requires much less delta- $\mathrm{V}$ to maintain, and will minimize the impact of thruster contamination on the instruments while also increasing the reliability of orbit determination for both science planning and subsequent data analysis. Orbits of this type can be maintained for 100 days with a more reasonable delta-V budget of $150 \mathrm{~m} / \mathrm{s}$.

Aside from propellant, the mission is also constrained in terms of power and communications downlink. Current mission designs comply with these constraints and still satisfy the baseline mission requirements. For communications, a low equatorial orbit has no view of Earth for approximately half of each orbit. In addition, the use of a single ground station at the White Sands Complex (WSC) can further restrict viewing opportunities. The baseline plan is to use either the Deep Space Network (DSN) or the Universal Space Network (USN) to supplement WSC. ,Those resources are shared among other assets, however, so availability is not guaranteed. Using just WSC, the spacecraft would be out of view for approximately half of the orbits in a given day.

To help manage these constraints, the operations phase will be defined on a "per orbit" basis. A $50 \mathrm{~km}$ orbit would take around 113 minutes. At 113 minutes per orbit, there will be approximately 12 to 13 orbits per day (several 12-orbit days followed by a "leap" day with 13 orbits). A variety of orbit "types" has been defined, and each orbit will be assigned one of these types. For example, one orbit type might be "NMS 1", which runs the Neutral Mass Spectrometer (NMS) in its first mode, which keeps the instrument continually oriented towards the positive velocity vector. The NMS 1 orbit might be followed by an "UVS" orbit where the Ultra Violet Spectrometer (UVS) will run. There may also be orbits dedicated to power regeneration and/or communications in which no science instruments are run. Table 1 shows a sample "day" comprised of these operational orbit types.

Table 1. Example 23.3-Hour Science Period

\begin{tabular}{|c|c|c|c|}
\hline Orbit & \begin{tabular}{|l} 
Orbit \\
Type
\end{tabular} & Instruments & Notes \\
\hline 1 & NMS (1) & NMS, DD & Rotating $360 \mathrm{deg} /$ orbit \\
\hline 2 & UVS (1) & UVS & Rotating $360 \mathrm{deg} /$ orbit \\
\hline 3 & NMS (1) & NMS, DD & Rotating $360 \mathrm{deg} /$ orbit \\
\hline 4 & Power & 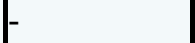 & Rotating $360 \mathrm{deg} /$ orbit \\
\hline 5 & UVS (2) & UVS & $\begin{array}{l}\text { Rotating } 360 \mathrm{deg} / \text { orbit } \\
\text { (w/ occult. })\end{array}$ \\
\hline 6 & NMS (2) & NMS & $\begin{array}{l}\text { Rotating 1 RPM } \\
\text { (rotisserie mode) }\end{array}$ \\
\hline 7 & UVS (2) & UVS & $\begin{array}{l}\text { Rotating } 360 \mathrm{deg} / \text { orbit } \\
\text { (w/ occult.) }\end{array}$ \\
\hline 8 & Comms & Radio & $\begin{array}{l}\text { Earth Pointing } \\
\text { (White Sands in view) }\end{array}$ \\
\hline 9 & NMS (2) & NMS & $\begin{array}{l}\text { Rotating 1 RPM } \\
\text { (rotisserie mode) }\end{array}$ \\
\hline 10 & UVS (1) & UVS & Rotating $360 \mathrm{deg} /$ orbit \\
\hline
\end{tabular}




\begin{tabular}{|l|l|l|l|}
\hline 11 & NMS (1) & NMS, DD & Rotating 360 deg/orbit \\
\hline 12 & Comms & Radio & $\begin{array}{l}\text { Earth Pointing } \\
\text { (White Sands in view) }\end{array}$ \\
\hline
\end{tabular}

During the Science Phase, LADEE will be in low lunar equatorial orbit, which is very unstable. The nominal mission plan may require orbit adjustments as often as two days apart for some periods of the mission. If through some contingence, the orbital maintenance adjustment were not carried out on schedule, the orbit could continue to decay to the point where impact would occur in two to three days after the missed maneuvers. In the case of missing a scheduled orbit maintenance maneuver, a contingency maneuver may be necessary to raise the periapsis altitude either to return to scheduled maintenance maneuvers or simply to prevent premature impact. In such cases, use of extra propellant would be justified but would probably shorten or change the rest of the mission.

\section{Orbit Maintenance}

As mentioned previously, the science requirement for lowaltitude conflicts with the propellant cost of maintaining a $50 \mathrm{~km}$ altitude. The rapid decay of the $50 \times 50 \mathrm{~km}$ circular science orbit will result in a large number of propulsive orbit maintenance maneuvers (OMMs). To minimize the expenditure of propellant, the orbit maintenance strategy will take advantage of gravitational perturbations, by using elliptical orbits up to $20 \times 100 \mathrm{~km}$. Orbit maintenance maneuvers in the science phase may be as often as every three to five days or as infrequently as once every two weeks. The various gravitational effects on the spacecraft will vary over the course of the lunar cycle.

Orbit maintenance maneuvers will require the most attitude changes: take out the slow spin, slew to align the thrust vector with the velocity delta, fire the OCS, return to nominal attitude, and resume the slow spin. In the UVS2 orbit type, the spacecraft may have an occasional slew above and below the limb mode viewing direction during sunrise or sunset terminator crossings in order to see dust "edge on" and increase the odds of detection.

\section{Momentum Management}

Desaturation events will occur as necessary, probably every few days. Momentum dumps are very short in duration and can be scheduled for compatibility with other operations.

\section{End-of-life termination}

Once the science operations deplete the onboard propellant, the orbit will be allowed to decay and the spacecraft will impact the lunar surface for disposal. Science data collection is an absolute priority during end-of-life maneuvers, given the opportunity for ultra-low-altitude observations. Priority will be given to ensuring a communications pass prior to impact in order to transmit these data.

\section{SPACECRAFT DESIGN}

The LADEE spacecraft bus design, derived from the Modular Common Spacecraft Bus architecture, is a small, low-cost spacecraft architecture designed to deliver scientifically and technically useful payloads to a variety of locations including lunar orbit. The spacecraft bus is a modular bus design that can be configured for a variety of mission objectives. Figure 2 shows the spacecraft bus in the LADEE configuration, with the instruments attached.

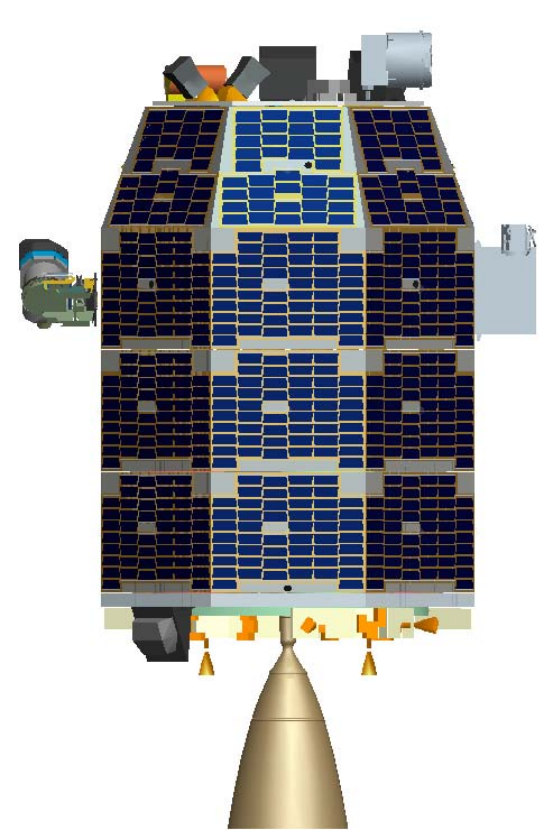

Figure 2: The LADEE Orbiter 


\section{Modular Common Bus Project Guidelines}

Since the Common Bus was designed to accomplish a variety of missions, it was designed independently of any particular payload. The original development guidelines are listed below:

SSO-1: Develop missions with destinations within the inner solar system, with destinations such as lunar orbit, lunar surface, earth-moon lagrange points, and near earth objects (NEOs).

SSO-2: Develop low-cost missions. This includes costs for small spacecraft development, launch vehicle, mission specific services/integration, instruments, operations, \& reserves.

SSO-3: Utilize cost effective launch vehicles and launch opportunities (e.g., Minotaur V, ESPA).

SSO-4: Develop missions within a short $(<36$-month) period.

SSO-5: Use a "Design-to-Capability" approach.

SSO-6: Manage projects as a NASA Category III, Risk Classification D Mission.

SSO-7: Use the following priority in hardware selection:

A) Existing hardware with space flight history

B) Existing hardware designed for space flight

C) Appropriate commercial-off-the-shelf (COTS) hardware designed for space

D) New design

SSO-8: Utilize technologies developed by other government technology programs.

SSO-9: Develop and operate multiple simultaneous projects that are appropriately phased.

\section{Design Overview}

The spacecraft bus is a lightweight carbon composite structure designed to accommodate launch loads and provide attenuation of impact loads. It is also designed for ease of manufacturing and assembly. The modularity of the design is intended not only for multiple mission configurations but also parallelism in development and assembly. The system-level components were drawn from low-cost flight-proven product lines.

For LADEE, the spacecraft bus modules consist of: (1) the Radiator Module, which carries the avionics, electrical system, and attitude sensors, (2) the Bus Module, (3) the Payload Module, which carries the two largest instruments, (4) the Extension Module, which houses the propulsion system, and (5) the Propulsion Module. The bus design is shown schematically in Figure 3.
One prominent characteristic of the bus design is that the solar arrays are body-mounted and fixed. While this arrangement reduces the available power, it eliminates deployment and articulation mechanisms, which eliminates several failure modes. It also ensures power production in almost any attitude, which enables very robust safe modes. This process also allows spacecraft attitude to be used for thermal control, and eliminates a hot and a cold side, which minimizes the need for heater power. The spacecraft can be flown in either a spinner or 3-axis control mode.

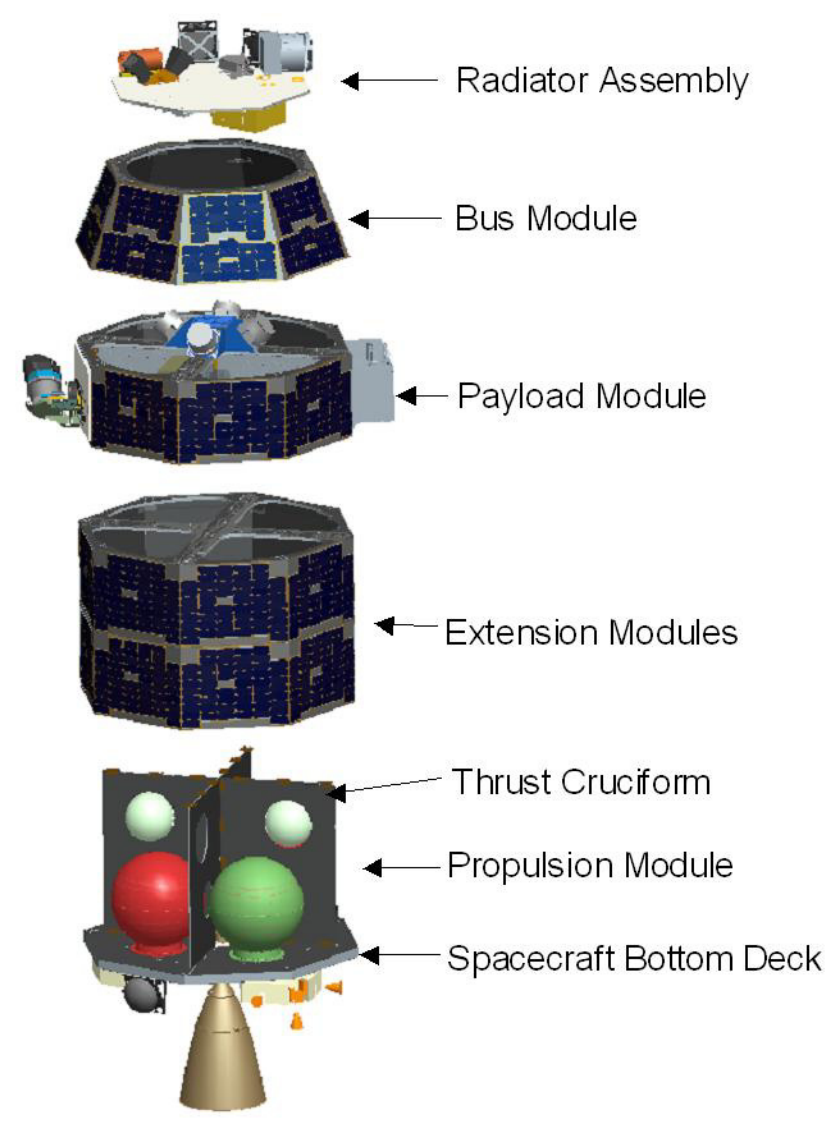

Figure 3: LADEE Bus Modules

System Diagram

Figure 4 shows the LADEE system block diagram. The flight avionics packages consists of a commercially available 8-slot $3 \mathrm{U}$ cPCI integrated avionics system providing the following functions:

- Command \& Data Handling Avionics

- Power Distribution

- Solar Array and Battery Charge Management

- Pyrotechnic Actuation

A separate electronics box in the Propulsion Module handles the valve driver actuation. The power system consists of an array of body-fixed solar panels, connected to batteries through the solar array control card within the avionics chassis. The body-fixed array design minimizes 


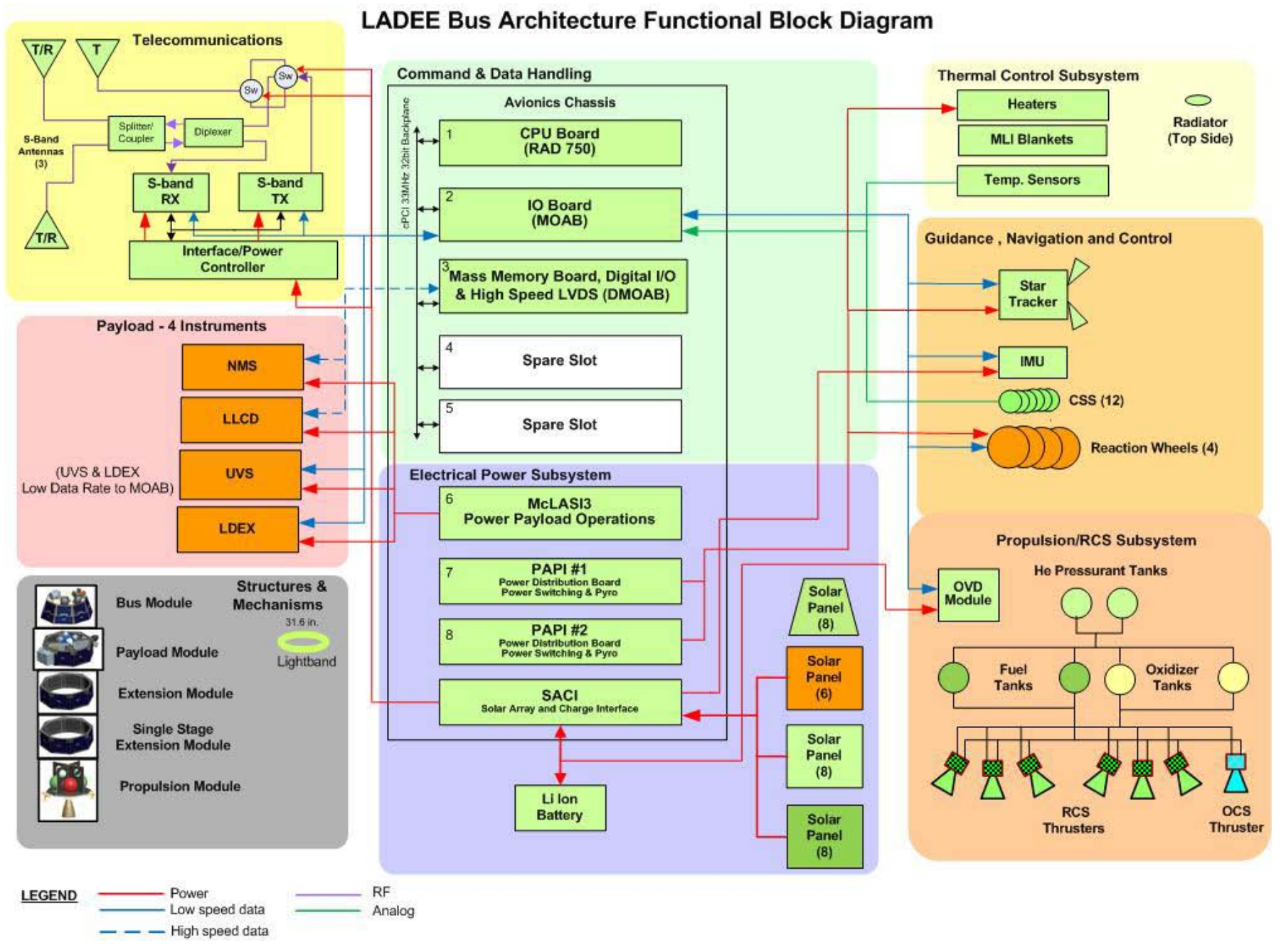

Figure 4: LADEE System Block Diagram

articulation on the spacecraft bus. The propulsion system is still being designed, but the baseline bipropellant propulsion system consists of a main thruster, six attitude control thrusters, two fuel tanks, two oxidizer tanks, two pressurant tanks, an ordnance valve driver box, and associated tubing and cabling. The spacecraft bus communications system consists of a modern architecture and modular design. The radio has separate receiver, transmitter and HPA modules and produces 5 Watts of RF transmitter power with flexible transmission power modes.

The spacecraft is using an evolved Omnidirectional/Medium Gain antenna design, developed at Ames, to achieve omni-directional coverage with a smaller area of medium gain response.

The avionics, batteries, attitude sensors, and two of the smaller science instruments are integrated into the Radiator Assembly (see figures 5 and 6). This arrangement

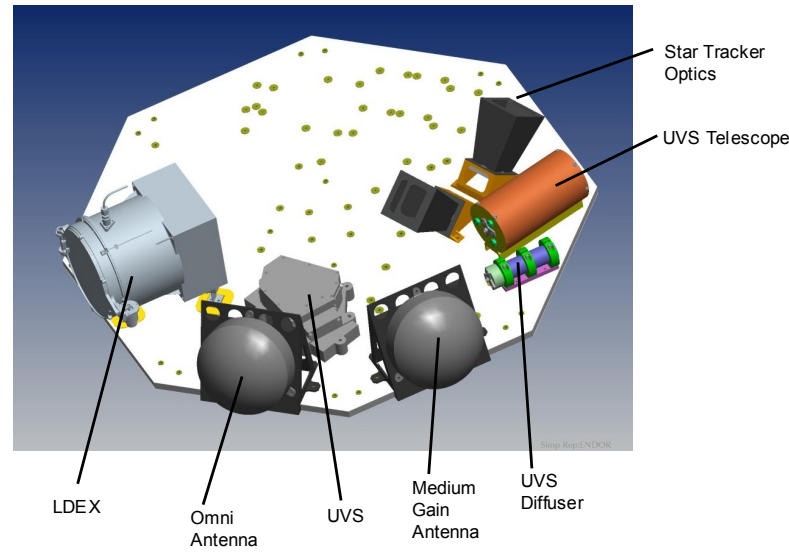

Figure 5: Radiator Assembly Top View 


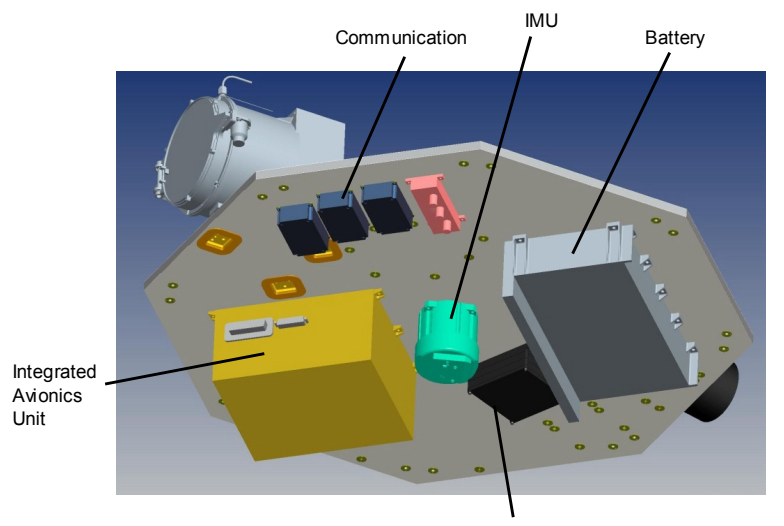

Figure 6: Radiator Assembly Bottom View

The two larger instruments (NMS and LLCD) are mounted on the Payload Module on opposite sides to balance the C.G. The reaction wheels, in a pyramid configuration, are also attached to the Payload Module. Figure 7 shows the layout of the Payload Module.

\section{Mission System ArChitecture}

The ground system is composed of the ground-based elements dispersed across the NASA centers and facilities. The LADEE Mission will utilize the Mission Operations

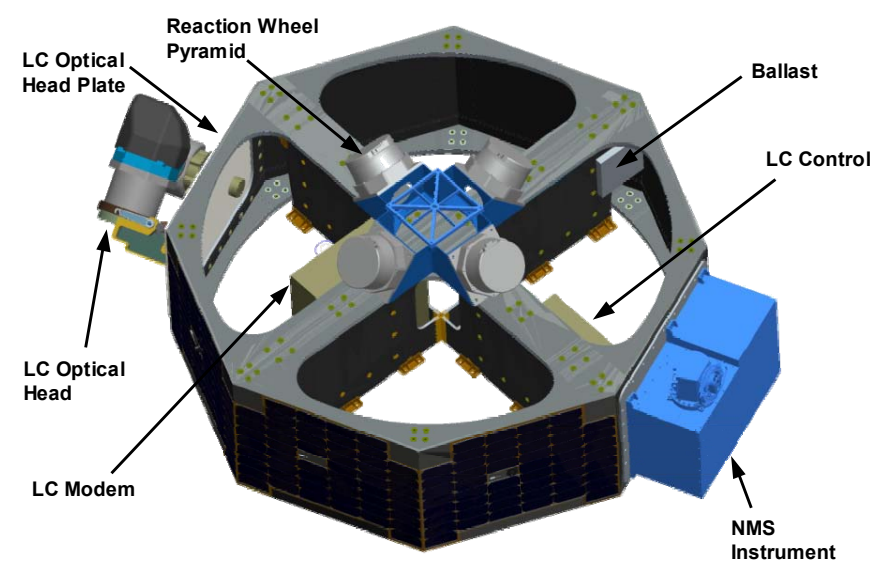

Figure 7: Payload Module Configuration

Center (MOC) located at NASA Ames Research Center (ARC) in Moffett Field, California. The project will use the Science Operations Center (SOC) located at Goddard Space Flight Center (GSFC) in Greenbelt, MD. The primary ground station will be located at the White Sands Complex (WSC) near Las Cruces, New Mexico.

The data from the ground station(s) will be routed to the MOC at ARC for processing, distribution and data storage/archiving. Science and instrument data, along with processed spacecraft health and safety data, will be transmitted to the GSFC SOC. The SOC will perform instrument data processing and scientific analysis of instrument data. Figure 8 shows a schematic representation of the Mission System Architecture and command and data flow within it.

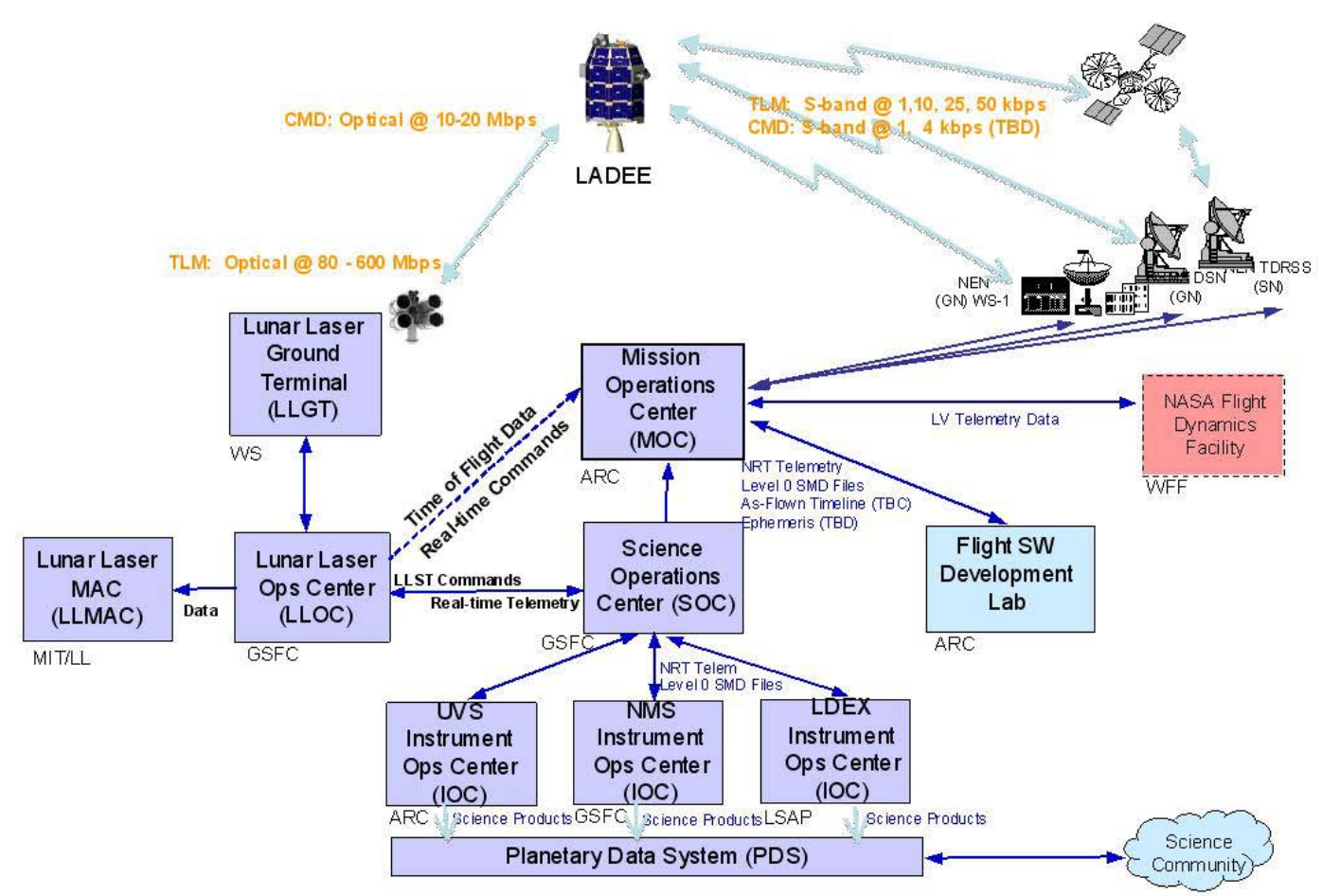

Figure 8: The Mission System Architecture showing data flow paths 

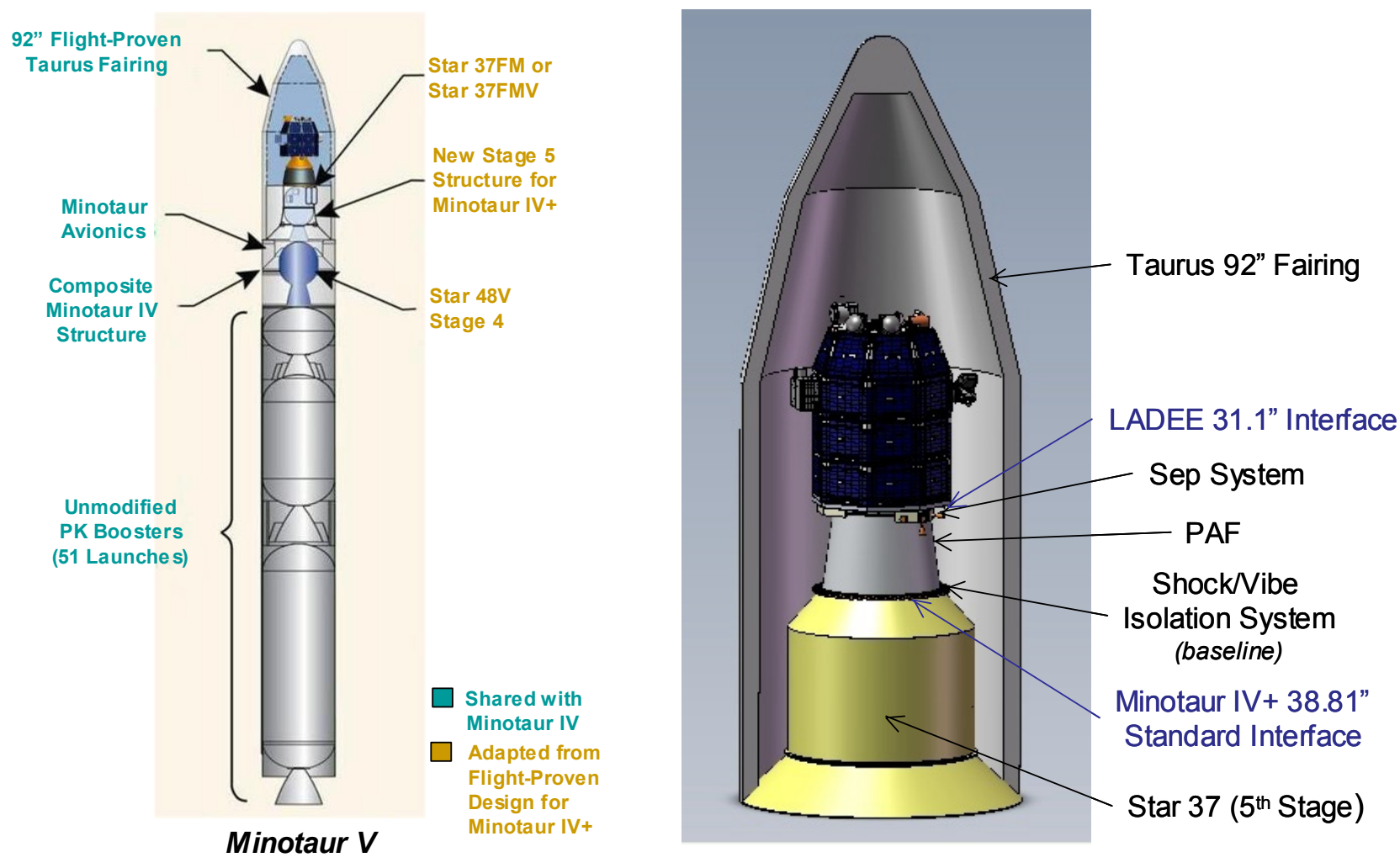

Figure 9: Minotaur V, with LADEE depicted in the fairing

\section{LAUNCH VEHICLE}

The LADEE mission will use a Minotaur V launch vehicle with WFF as the launch site (Pad 0B). The expected launch date is late 2012. The Minotaur V is a new launch vehicle, and is evolved from the Minotaur IV design. It includes a new 3-4 interstage, new stage 5 avionics cylinder, stage 5 spin/de-spin, PAF, and the STAR 37 motor. The first three stages of the Minotaur IV, in turn, are based on the highly successful Peacekeeper Missile. The LADEE spacecraft is attached to the Star 37 fifth stage using 38-inch to 31-inch PAF. Figure 9 shows the launch vehicle stack and the LADEE configuration within the fairing.

\section{SUMMARY}

LADEE is an important component in NASA's portfolio of near-term lunar missions, addressing objectives that are currently not covered by other U.S. or international efforts. Its observations must be conducted before large-scale human or robotic activities irrevocably perturb the tenuous and fragile lunar atmosphere. LADEE will also demonstrate the effectiveness of a low-cost, rapid-development program, utilizing a modular bus design launched on the new Minotaur V launch vehicle. Once proven, this capability could enable future lunar missions in a highly cost constrained environment.

\section{REFERENCES}

[1] The Scientific Context for EXPLORATION of the MOON, Space Studies Board, Division on Engineering and Physical Sciences, National Research Council of the National Academies, The National Academies Press, Washington, D.C., 2007

[2] Caffrey et al., Developing the Payloads for the Lunar Atmosphere and Dust Environment Explorer (LADEE) Mission, 2010 IEEE Aerospace Conference, Big Sky, Montana, March 6-13, 2010.

[3] William S. Marshall, Mark F. Turner, Butler P. Hine, and Alan R. Weston, Small Spacecraft in Support of the Lunar Exploration Program, White-paper, 2007.

\section{BIOGRAPHY}

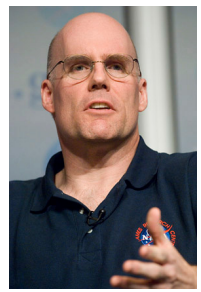

Butler Hine is project manager for the LADEE mission. Prior to LADEE, Dr. Hine managed the Small Spacecraft Division at NASA Ames Research Center, which developed ways to build low-cost, high-performance spacecraft to enable future NASA missions. 